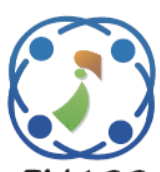

\title{
Integrating Particle Swarm Optimization and Routh-Hurwitz's Theory for Controlling Grid-Connected LCL-Filter Converter
}

\author{
Fadhil A. Hasan $^{1 *} \quad$ Lina J. Rashad $^{1} \quad$ Abdulrahim T. Humod $^{1}$ \\ ${ }^{1}$ Department of Electrical Engineering, University of Technology, Baghdad, Iraq \\ * Corresponding author's Email: 30077@uotechnology.edu.iq
}

\begin{abstract}
This paper proposes a method of integrating the Particle Swarm Optimization (PSO) technique with Routh-Hurwitz's stability theory in a special optimizing process as an effective tuning method for the PI controller. This overcomes the problem of resonance frequency between the grid and filter, which affects on system's stability margin. This concept realised by combining multiple restrictions functional, time and frequency behaviour besides the stability criterion, as a comprehensive evaluation to enhance the performance and its activity. The decoupled model of the LCL-filter grid-connected voltage source inverter (VSI) is used to simulate the multi-loop current control scheme. This approach operates in offline mode to achieve the optimal controller's parameters. Simulation results demonstrate an interesting harmony between the system's performance and stability in different operating conditions, which gives phase margin $=87^{\circ}$, gain margin $=7 \mathrm{~dB}$, and damping ratio $=0.55$. The proposed design shows superior performance, as compared with previous works, in dynamic and steady state performances, which gives rise time $=7.861 \mathrm{~ms}$, settling time $=27.6 \mathrm{~ms}$, overshoot $=2.282 \%, \mathrm{THD}=1.967 \%$, and $\mathrm{RF}=3.017 \%$, besides an acceptable current's harmonics contents.
\end{abstract}

Keywords: Power grids, Control design, PI control, Distributed power generation, Particle swarm optimization.

\section{Introduction}

Recently, the power production manufacturers are recommended to depart toward the distributed generations (DGs) systems, because of their advantages such as; greenhouse gas emissions and reliability, as well as to cost rising of the traditional energy. The DGs units are utilized as an integrating system connected to the low voltage distributed system to deliver the renewable green power such as; fuel cell, PV, or wind energies to the power grid utilizing power coupling converter [1]. The voltage source converter (VSI) is used to control the active and reactive powers injected, as demand, through the grid-connection DG system. The pulse width modulation technique of the VSI tends to undesired low, and high-order current harmonics injected to the utility system $[2,3]$. Therefore, an LCL filter is implemented as an interfacing network between the VSI and the power grid. The LCL filter topology is more effective from the $\mathrm{L}$ filter in the reduction of current's harmonics and improves the total harmonic distortion (THD). The active damping can also be used with an LCL-filtered grid-connected inverter. Compared to the L filter, the LCL filter has better attenuation of the switching frequency harmonics, yielding lower size and costs [4]. There are many extensive researches, in the literature, related to the controller's performance in term of system stability, resonance impedance. The literature has extensively researched the effectiveness of various control methods in respect to their reliability, resonance impedance, and durability to parameters variants. To suppress low-order harmonics of the network current caused by phase looked loop (PLL) perturbations under weak grid conditions, a feedforward control method is used to compensate PLL perturbations and revise the output impedance [6]. A hybrid system consists of a static VAR compensator (SVC) in parallel with a capacitive- 
coupling grid-connected inverter (CGCI). [7]. The LCL filter circuit can increase model order, but there is a resonance peak, which can affect system stability. To suppress the resonance harmonics, the most effective approach is to use passive damping consisting of series or parallel resistance with the filter capacitance [8]. The serial branches decrease losses but reduce the damping ability of high-order harmonics. However, the parallel branch has no impact on the low and high-order harmonics, but it increases the losses.

Traditionally, a simple L filter can fulfil this function, but a large inductance usually needs to be adopted, which is costly, bulky, and inefficient. Therefore, higher-order power filters, such as LCL, LLCL, LTL, LTCL, etc., have been widely investigated due to their better harmonic attenuation ability with lower total inductance [9].

The predictive current control, modifying the sampling time, or the PWM method can be used to enhance the controller's robustness against grid impedance variation caused by inherent control delay [10]. The state feedback controller method simulating virtual resistance to achieve system damping, results prove the effectiveness of the virtual resistance to suppress the resonance harmonics. [11]

A master-slave current control is used for the LCL-filter-based grid-connected inverter operating under variable grid condition. This system is composed of a grid-connected voltage-source inverter (GC-VSI) in series with a bidirectional voltage source converter (BVSC) at the inverter output. The GC-VSI is responsible for line current regulation, while the B-VSC takes charge of harmonic current suppression and grid impedance cancellation [12]. A hybrid current control scheme, utilizing the decoupled predictive hysteresis capacitor current controller integrated with the linear grid current controller, is used by applying the $60^{\circ}$ discontinuous pulse-width modulation scheme and using the virtual dual-buck decoupled structure [13]. The proportional-integral (PI) controller is the most commonly used, owing to its ease of implementation. Therefore, aims at comparing the operation of the PI controller with its stationary reference frame equivalent known as the proportional-resonant (PR) controller. Both controllers are used in the voltage oriented control scheme for a three-phase converter [14]. Even so, such enhanced techniques make the system being sophisticated, complex, and system stability is difficult to control.

The most challenge that needs to be addressed, in designing the PI current controller, is to minimize the complex coupled terms between the d-axis and q-axis models in the synchronously reference frame. Decoupling method with ADRC can remove the coupled channels of controlled system with multiple inputs and multiple outputs, and it can improve the robustness for LCL-type to reject internal and external disturbances [15]. The design of a PI-based grid current vector control system for a three-level GCVSC with an actively damped LCL output filter is used, particularly focusing on practical issues to max out control performance as well as taking requirements concerning current harmonics into account[16]. In the rotating reference frame, there is a strong coupling between $\mathrm{d}$ axis components and $\mathrm{q}$ axis components, which seriously affects the performances of LCL-type grid-connected converters. If the coupling terms are ignored as external disturbances, the independent control of the $\mathrm{d}$ axis components and $\mathrm{q}$ axis components can be realized in traditional current control schemes. However, this causes modelling distortion and reduces the performances of grid-current. By analyzing the transfer function of grid-connected converters, coupling compensation terms are introduced in a current control scheme [17, 18].

The tuning of the PI controller is accomplished utilizing the zero/pole placement in the s plane, which intends to obtain a best adjustment between the high dynamic performance of the dc output voltage and the reduction of the ac current overshoot [19]. The particle swarm optimization (PSO) technique is presented in [20] to optimize the $\mathrm{K}_{\mathrm{p}}$ and $\mathrm{K}_{\mathrm{i}}$ parameters of the PI controller of the DC-link voltage. The objective fitness function is evaluated the ripple factor and the THD of the injected current. Whilst, the PSO is utilized, in [21], to design a nondiagonal state cost matrix. The diagonal cost matrices are designed such that the requirements are met at multiple loading conditions.

Unfortunately, all previous works in the literature were utilized only a single view of consideration such as ripple factor, THD, time response, frequency response, etc.. Unlike those works, this paper proposed an adequate intelligent procedure for optimizing the PI controller of the outer-loop, which combines the time and frequency constraints besides Routh-Hurwitz's stability criteria. The proposed design procedure integrates those various restrictions to formulate an efficient fitness function (FF) that utilized in the particle swarm optimization (PSO) algorithm. The effectiveness of the proposed method relies on this FF which presents a multi-objective constraint represents a comprehensive evaluation of the system performance. According to performance requirements, the presented optimization method 
involves a convergence to target values of the time and frequency (phase and gain margins, crossfrequency, overshoot, steady-state error), besides stability constraints. This approach distinguishes this technique from previous works, in controller adjustment based on the PSO technique, as controller having a theoretical certification for effectiveness and stability for closed-loop systems. The method presents an offline optimization process.

This paper organized as follows: in section 2 the mathematical representation of the LCL-filter interfaces between VSI and the grid. The direct decoupling approach is used to obtain the overall transfer function. Sections $3 \& 4$ represent an overview of the PSO technique and how to fabricate the objective FF. section 5 represents the optimization and simulation results and compare them with previous works.

\section{VSI-LCL mathematical model}

The typical utilized scheme of VSI-LCL system can be seen in Fig.1. The stationary frame analysis of the network can be expressed, using Kirchhoff's voltage law, as follows:

$$
\left\{\begin{array}{l}
\tilde{v}_{1}-\tilde{v}_{c}=L_{1} p \tilde{l}_{1}+r_{1} \tilde{l}_{1} \\
\tilde{l}_{c}=C p \tilde{v}_{c} \\
\tilde{v}_{c}-\tilde{e}=L_{2} p \tilde{l}_{2}+r_{2} \tilde{l}_{2} \\
\tilde{l}_{1}=\tilde{l}_{c}+\tilde{l}_{2} \\
L_{2}=L_{f}+L_{g} \\
r_{2}=r_{f}+r_{g}
\end{array}\right.
$$

Where the superscript $\sim$ represents the threephase ac quantities; $p$ is the derivative operator; $\tilde{v}_{1}$, $\tilde{l}_{1}$ is the converter side voltage and current; $\tilde{v}_{c}, \tilde{i}_{c}$ are the capacitance voltage and current; $\tilde{l}_{2}$ is the grid current; $L_{1}, L_{f}, L_{g}, r_{1}, r_{f}, r_{g}$ are the converter side filter, grid side filter, grid inductances and parasitic resistances respectively.

According to the concepts of the rotating reference frame that reduces the coupling effect, between the three-phase quantities, and complexity

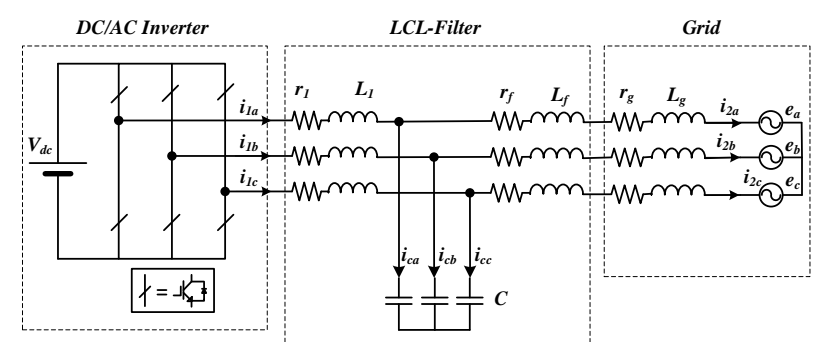

Figure. 1 Typical scheme of the VSI-LCL grid-connected system

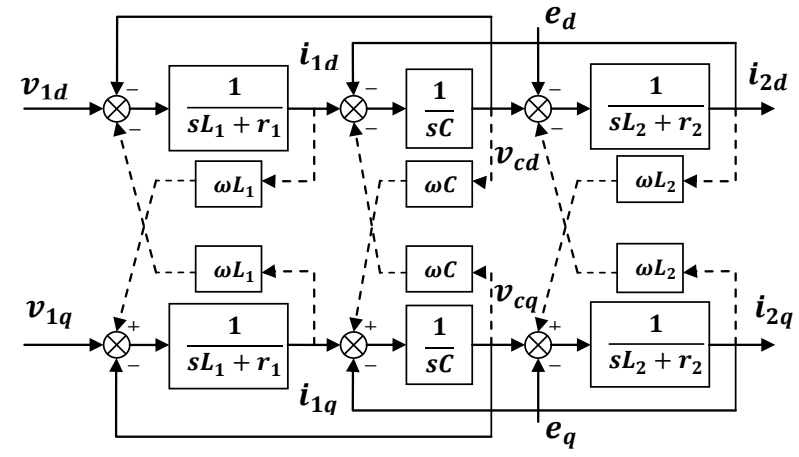

Figure. 2 The dq-model of VSI-LCL grid-connected system

the abc-dq transformation is used, the dq-axis equations can be expressed as follows:

$\left\{\begin{array}{l}v_{1 d q}-v_{c d q}=L_{1} p i_{1 d q}+r_{1} i_{1 d q}+\left[\begin{array}{cc}0 & L_{1} \omega \\ -L_{1} \omega & 0\end{array}\right]\left[\begin{array}{l}i_{1 d} \\ i_{1 q}\end{array}\right] \\ i_{1 d q}-i_{2 d q}=i_{c d q} \\ i_{c d q}=C p v_{c d q}+\left[\begin{array}{cc}0 & C \omega \\ -C \omega & 0\end{array}\right]\left[\begin{array}{c}v_{c q} \\ v_{c d}\end{array}\right] \\ v_{c d q}-e_{d q}=L_{2} p i_{2 d q}+r_{2} i_{2 d q}+\left[\begin{array}{cc}0 & L_{2} \omega \\ -L_{2} \omega & 0\end{array}\right]\left[\begin{array}{l}i_{2 d} \\ i_{2 q}\end{array}\right]\end{array}\right.$

Where the subscript $d$ and $q$ represent the dqaxis components; $\omega$ is the angular frequency of the utility, which obtained from a phase-locked loop (PLL) mechanism [22]. The synchronously reference frame scheme of the VSI-LCL system can be achieved from Eq. (2) as depicted in Fig. 2. Where, the dotted lines represent the coupling terms. It's quite the essential reason for the coupling issue. Which denotes that any fluctuations in the q-or dmodel causing variations in the conforming dqmodel. To attain the autonomous controlling of the injected active and reactive power, that effects generated via the coupling expressions must be eliminated.

Automated Controllers concept indicates that any extraneous coupling effect can be ignored directly in the transfer function solving approach. Thus, these coupling expressions among $\mathrm{d}$-axis and q-axis are cancelled straightly as external perturbation signals. According to this strategy the synchronously reference frame scheme of the VSILCL system can be illustrated as in Fig. 3.

Based on the previous assumption the overall transfer function can be defined of the plant and its parameters. A third-order transfer function from the converter output voltage $v_{1 d q}(s)$ to the grid-injected 
current $i_{2 d q}(s)$, valid for both d-axis and q-axis, is given by:
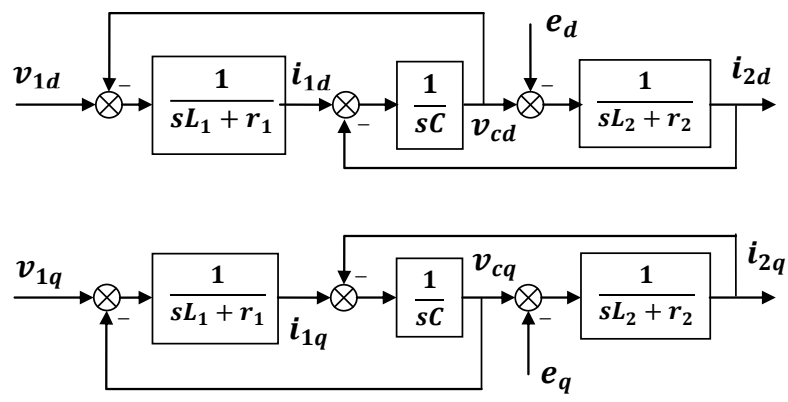

Figure. 3 The decoupled dq-model of the system

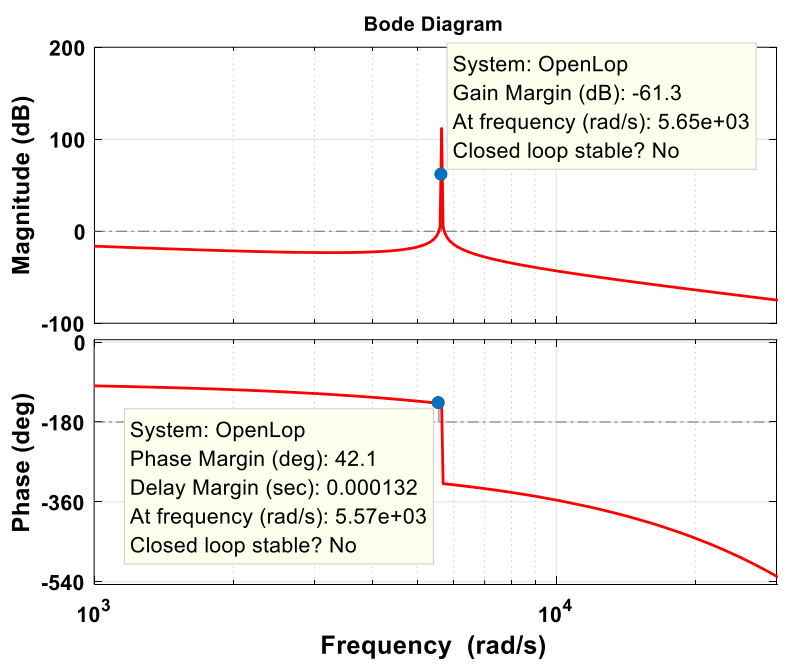

Figure. 4 The frequency response of the LCL-filter

$$
G_{d q}(s)=\frac{i_{2 d q}(s)}{v_{1 d q}(s)}=\frac{1}{a_{3} s^{3}+a_{2} s^{2}+a_{1} s+a_{0}}
$$

Where:

$$
\begin{aligned}
& a_{0}=r_{2}+r_{1}, \\
& a_{1}=L_{1}+L_{2}+C r_{1} r_{2}, \\
& a_{2}=C L_{1} r_{2}+C r_{1} L_{2}, \\
& a_{3}=C L_{1} L_{2} .
\end{aligned}
$$

The frequency response and the resonance limit can be investigated by means the bode-plot of this system as shown in Fig. 4.

\section{Current control strategy}

The current controller's layout of the presented model is debated in this section depends on the transfer function in (3). According to the Routh's stability criteria, the single closed-loop controller scheme is unstable [23]. The stability limits of the closed-loop system can be realized be means the root-locus trajectory as shown in Fig. 5. Therefore, an additional controller dedicated to preserving the system's stability must be presented. Different types of multi-loop controller strategies are frequently

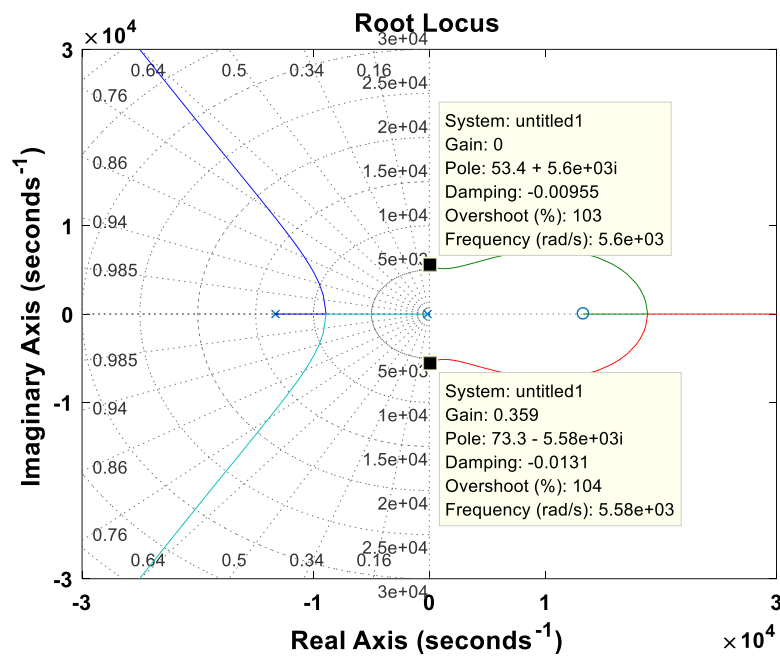

Figure.5 Root-locus of the closed-loop system

accomplished. The approach of multi-loop controlling inherently assorts virtual impedance to adapt the internal and external impedances. To defeat the restrictions of the single-loop controller method, the internal impedance, which is predominantly in charge of giving the required damping to the system, must be modified by inserting an inner controller.

Nevertheless, the adapting of the inner controller parameters is not simple. Considering two variables, converter side current and the capacitor current, are utilized as the inner loop parameters [23, 24]. A proportional $\left(K_{\text {inv }}\right)$ controller is used to regulate the capacitance current $i_{c}$, which represents the innerloop controller gain, is the assurance of stable performance. Practically $K_{i n v}$ includes the transfer function of the VSI which represents the modulation index of the PWM technique.

Typically, the achievement of a closed-loop control scheme is realized by using digital microcontrollers which includes analogue-to-digital (ADC) processing, computations, and PWM technique. These operations insert a time delay. The time delay $\left(T_{d}\right)$ depends on the switching frequency $\left(f_{s w}\right)$, which can be presented as follows [4]:

$$
G_{d}(s)=e^{-T_{d}} \quad, T_{d}=1.5 T_{s w}
$$

The influence of hardware delay time is considered for the inner-loop controller. The bodeplot of the opened-loop inner controller while subjected to various time delays is depicted in Fig. 6. The frequency response points that the stability of the system is limited at a small time delay.

Therefore, a proportional-integral (PI) controller, with proportional gain $K_{p}$ and integral gain $K_{i}$, is 
employed to regulate the grid side current $i_{2}$, which

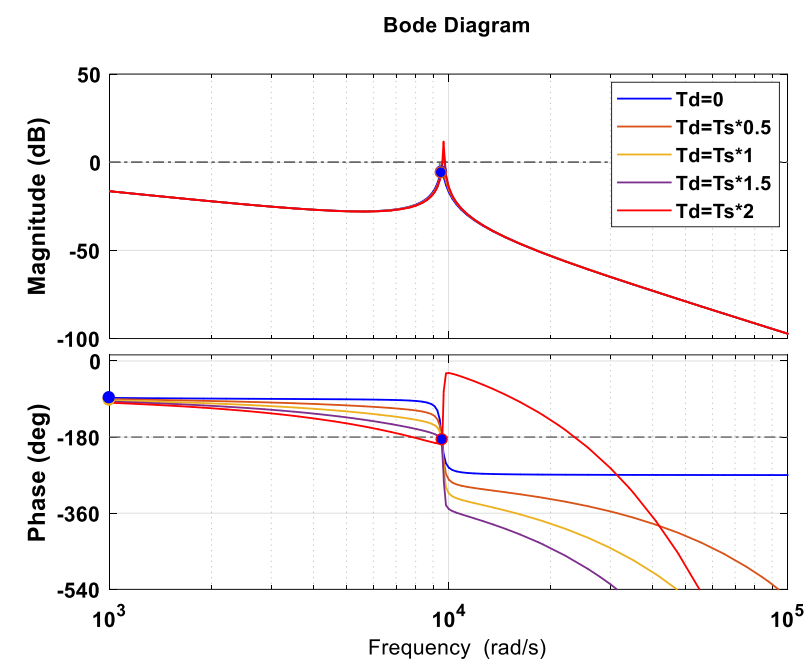

Figure.6 Time delay variation

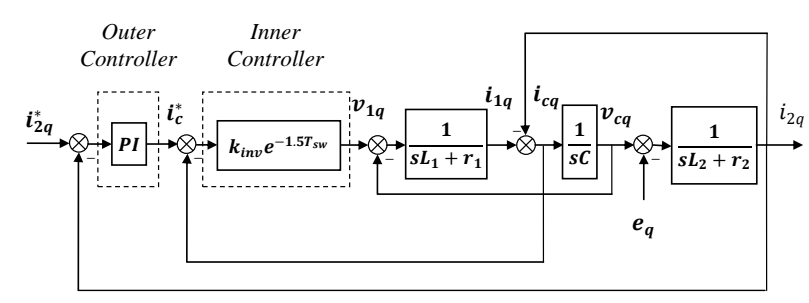

Figure.7 Current control scheme

represents the external loop controller parameter, which tracks the command current. This strategy is adopted in this work; the scheme of the dual-loop controller technique can be seen in Fig. 7.

Correspondingly, the open-loop transfer function of this dual-controller strategy is extracted as following:

$$
G_{\text {open }}(s)=\frac{f_{1} s+f_{0}}{g_{4} s^{4}+g_{3} s^{3}+g_{2} s^{2}+g_{1} s+g_{0}} e^{-T_{d}}
$$

Where:

$f_{0}=K_{i} K_{\text {inv }}$,

$f_{1}=K_{p} K_{\text {inv }}$,

$g_{0}=0$,

$g_{1}=r_{1}$,

$g_{2}=C r_{1} r_{2}+L_{1}+L_{2}$,

$g_{3}=C r_{1} L_{2}+C r_{2} L_{1}+C L_{2} K_{i n v}$,

$g_{4}=C L_{1} L_{2}$.

Consequently, the closed-loop transfer function of the compensated system can de expressed as follow:

$$
G_{\text {close }}(s)=\frac{f_{1} s+f_{0}}{m_{4} s^{4}+m_{3} s^{3}+m_{2} s^{2}+m_{1} s+m_{0}} e^{-T_{d}}
$$

Where:

$$
\begin{aligned}
& m_{0}=K_{i} K_{i n v} \\
& m_{1}=K_{p} K_{i n v}+r_{1} \\
& m_{2}=C r_{1} r_{2}+L_{1}+L_{2} \\
& m_{3}=C r_{1} L_{2}+C r_{2} L_{1}+C L_{2} K_{i n v} \\
& m_{4}=C L_{1} L_{2} .
\end{aligned}
$$

The selection of the controller's parameters $K_{p}$ and $K_{i}$ is not a straightforward operation, and many techniques are used in the literature concern on adapting controller parameters. Previous works have a common point is not exploring together time and frequency domain specifications for tuning the controller. In fact, there is an inherent relationship between concentrating parameters of the time and frequency domains (such, between overshoot and phase margin, or between steady-state error and low-frequency gain). Therefore, this work exploring together the time and frequency domain concentrates in tuning the controller's parameters, by using PSO algorithm. As the first step the boundaries of the searching space can be found by applying Routh's stability criteria to the characteristic equation of closed-loop controlled system. When these polynomial formulas are Hurwitz, then the closed-loop controller is stable for all variable parameters quantities.

The main goal of this paper is to obtain, offline, the PI controller's gains by realizing the following:

The performance of the closed-loop system fulfils the Routh's stability criteria. The Fitness Function (FF) represents both frequency and timedomain performance criteria (e.g. over-shoot, steady-state error, and phase and gain margins).

\section{Optimization technique}

The most powerful optimization technique is Particle swarm optimization (PSO), which presented by Kennedy and Eberhart. It was inspired by observing a simplistic social behavior and considered to be effective in resolving existing nonlinear optimization systems [25]. Within a faster computation time and reliable convergence properties, the PSO methodology generates a highquality solution among all precisely stochastic techniques $[20,21]$. Many studies remain underway to verify the capability of PSO in solving nonlinear optimization problems. Since the PSO technique is a brilliant optimizing approach and a promising strategy to addressing the issue of optimum PI 
controller gains; then, this work proposed a combining algorithm between PSO and Routh's theory for tuning the PI controllers. This methodology is evolved from swarm studies like fish schooling and bird flocks. It can be easily accomplished and features reliable convergence with precise computing efficiency [20].

Instead of implementing evolutionary processors to regulate the particles, as in other adaptive optimization methods, the particles in PSO fly in the search field with a trajectory that is continuously modified according to its travel experiences and the travel experiences of its partners. In the $k$-dimension search field, every particle is regarded as a volumeless particle. Every particle is continuously tracking of its location in the searching space correlated with the optimal solution accomplished so far, and this is named pbest. A further best evaluation recorded by the particle swarm optimizer's global version is the absolute best value, and its destination, achieved yet by any particle in the swarm, is named gbest.

The PSO algorithm involves adjusting that particle's position and velocity to its pbest and gbest at each stage. A stochastic variable weights the acceleration, producing different random numbers for acceleration to pbest and gbest positions.

For instance, the $j^{\text {th }}$ particle is expressed as $\left(x_{j, 1}, x_{j, 2}, \ldots, x_{j, k}\right)$ in the $k$-dimension field. The optimal prior location of the $j^{\text {th }}$ particle is registered and explained as pbest $=\left(\right.$ pbest $_{j, 1}, \ldots$, pbest $\left._{j, k}\right)$. The optimum particle among all of the particles in the swarm is indexed and expressed by the gbest $_{k}$. The rate of deviation in the particle's location (velocity) is expressed as $v_{j}=\left(v_{j, 1}, v_{j, 2}, \ldots, v_{j, k}\right)$. The updated velocity and location of each particle can be evaluated by utilizing the existing velocity and the distance from pbest ${ }_{j, k}$ to gbest $_{j, k}$ as in:

$$
\begin{aligned}
& v_{j, k}^{(t+1)} \\
& \left.=W \cdot v_{j, k}^{(t)}+c_{1} \text { rand }^{(}\right)\left(\text {pbest }_{j, k}-x_{j, k}^{(t)}\right) \\
& +\quad c_{2} \text { Rand }_{(}\left(\text {gbest }_{j, k}-x_{j, k}^{(t)}\right)
\end{aligned}
$$

$x_{j, k}^{(t+1)}=x_{j, k}^{(t)}+v_{j, k}^{(t+1)}$

$j=1,2, \ldots$, no. of particles

$k=1,2, \ldots$, no. of dimentions

Where, $t$ is the iteration pointer, $v$ is the velocity of particle, $W$ inertia weight, $c_{1}$ and $c_{2}$ are acceleration constants, rand ( ), Rand( ) are random values between 1-0, pbest local best particle, gbest global best particle.

The coefficients $c_{1}$ and $c_{2}$ comprise the relative weight of probabilistic acceleration aspects that drag each particle to pbest and gbest locations. Small values enable particles to travel away from target zones until being withdrawn. Furthermore, large values lead to a rapid motion to, or previous, target zones. As per previous researches, the acceleration constants $c_{1}$ and $c_{2}$ were sometimes put to be (1-2). Adequate choice of inertia weight $W$ in (6) creates a compromise between global and local discovery, allowing reduced iteration on aggregate to locate a reasonably optimum solution. As initially improved, $W$ frequently declines sequentially from about 0.9 to 0.4 through the runtime. Notably, the inertia weight $W$ is expressed as shown by [25]:

$W=W_{\max }-\frac{W_{\max }-W_{\min }}{\text { iter }_{\max }} \times i$ iter

Where, iter $_{\max }$ is the maximum number of iterations, iter is the current iteration.

\section{Objectives fitness function}

This system contains two controllers (inner and outer), a multi-objective function returns a value obtained by summing three expressions; $\alpha\left(x_{j, k}\right)$, $\eta\left(x_{j, k}\right)$, and $\delta\left(x_{j, k}\right)$ which evaluates the degree of fitness of the system performance for the individual control loop. The three functions represent the time and frequency domain responses as well as the Routh-Hurwitz's stability criteria.

The first expression $\alpha\left(x_{j, k}\right)$ can be evaluated as:

$$
\begin{aligned}
\alpha\left(x_{j, k}\right)=\min & \left(\left|\frac{M_{p}^{*}-M_{p j}\left(x_{j, k}\right)}{M_{p}^{*}}\right|\right. \\
& \left.+\left|\frac{\omega_{c r}^{*}-\omega_{c r j}\left(x_{j, k}\right)}{\omega_{c r}^{*}}\right|\right)
\end{aligned}
$$

Where $M_{p}^{*}$ and $\omega_{c r}^{*}$ are the desired phase margin and crossover frequency. The objective function measures the worst difference between the desired values and the current values of each particle. By using MATLAB software, one can find $M_{p j}\left(x_{j, k}\right)$ and $\omega_{c r j}\left(x_{j, k}\right)$ during the optimization process.

In this manner, the minimization of $\alpha\left(x_{j, k}\right)$ does not guarantee for enhancing the frequency and time responses. Thus, to improve the limited performance boundaries of the gain margin, overshoot and 
steady-state error are added to the $\mathrm{FF}$ as an objection flag $\eta\left(x_{j, k}\right)$.

These criteria can be formalized as a (if then) statement:

$$
\left\{\begin{array}{l}
\text { If }\left(M_{g j} \geq \widehat{M_{g}} \text { and } O_{s h j} \leq \widehat{O_{s h}} \text { and }\left|e_{s s j}\right| \leq \widehat{e_{s S}} \ldots\right. \\
\text { Then } \left.\eta\left(x_{j, k}\right)=1 \quad \text {.. and } \widehat{e_{s S}} \text { and }\left|u_{c}\right| \leq \widehat{u_{c}}\right) \\
\text { Else } \eta\left(x_{j, k}\right)=10^{8}
\end{array}\right.
$$

Where $\overline{M_{g}}, \widehat{O_{s h}}, \widehat{e_{s S}}$ and $\widehat{u_{c}}$ are the lower boundary of the gain margin, the upper boundaries of overshoot and steady-state error and maximum control signal respectively.

The third part of the fitness function $\delta\left(x_{j, k}\right)$, represents the degree of robustness obtained according to Routh-Hurwitz's criteria. This also represents an objection flag and can be implemented as a (if - then)statement:

$$
\left\{\begin{array}{l}
\text { If (the closed-loop char. Equations is Hurwitz) } \\
\text { Then } \delta\left(x_{j, k}\right)=1 \\
\text { Else } \delta\left(x_{j, k}\right)=10^{8}
\end{array}\right.
$$

Combining these three functions, the overall fitness function can be formed as:

$$
F\left(x_{j, k}\right)=\alpha\left(x_{j, k}\right) \eta\left(x_{j, k}\right) \delta\left(x_{j, k}\right)
$$

Minimizing $\alpha\left(x_{j, k}\right)$ is relevant to obtaining controllers which contribute to open-loop responses with phase margins and crossover frequencies closer to reference values; $\eta\left(x_{j, k}\right)$ is referred to having controllers which conform within gain margin, overshoot, and steady-state error constraints by rejecting all particles violate these criteria; $\delta\left(x_{j, k}\right)$ is attributed to getting controllers that maintain the closed-loop within Routh-Hurwitz's stability. These factors can easily be calculated by using MATLAB codes. The boundaries can be chosen to give adequate performance for each controller, such as [e.g. $\bar{M}_{g}=10 \mathrm{~dB}, M_{p}^{*}=60^{\circ}, \omega_{c r}^{*}=300 \mathrm{rad} / \mathrm{s}$, $\widehat{O_{s h}}=2 \%, \widehat{e_{S S}}=2 \%$ and $\widehat{u_{c}}=1.5$ p.u.].

The first step in the procedure is to identify the plant parameters and transfer function. Secondly, the inner and outer controllers are selected and the closed-loop characteristic equation is expressed. The third step is to find the searching domain boundaries according to Hurwitz's criteria. The fourth step is to starting the PSO algorithm to find the optimum parameters of the controllers, which find out by evaluating the fitness function in (12). The criteria of Hurwitz's stability are investigated for each particle in the searching domain. This process sequence is repeated in an offline manner for each controller (inner and outer) individually.

\section{Simulation result}

The proposed controller design strategy is simulated by using MATLAB-SIMULINK and MFile. The double-loop controller scheme is implemented with direct decoupling model as mentioned before. The PSO algorithm is used to search for the optimum controller's parameters by exerting a particular objective fitness function. The goal of the optimization process is to minimize the objective fitness function, which represents a multiobjective optimization criterion. A combination of the time and frequency specifications, as well as Routh-Hurwitz's stability criteria, develops the optimization fitness function. The algorithm process is executed in offline evaluation for each (d) and (q) controller separately. Table 1 shows the parameters of the LCL-filter and the utility grid.

The particle swarm optimization algorithm is initialized by using the following parameters: $\mathrm{N}=50$ birds, 100 epochs, $c_{1}=1.3, c_{2}=1.3, W_{\text {initial }}=0.7$, $W_{\max }=0.9, W_{\min }=0.4$.

Assuming $k_{i n v}=1$, the obtained optimum values of the PI controller gains are: $\left(K_{p}=\right.$ 1.597, $\left.K_{i}=92.708\right)$. Fig. 8 shows the tendency of particles moving within the searching field toward the global optimal solution, which tracking the corresponding particles of optimum solution (the shown particles are part of the total particles). The solid red line depicts the path followed by the particles toward the global optima.

Table 1. Parameters of the grid-connected converter

\begin{tabular}{|l|c|}
\hline \multicolumn{1}{|c|}{ Parameter } & Value \\
\hline Switching frequency fsw & $10 \mathrm{kHz}$ \\
\hline Sampling frequency fs & $20 \mathrm{kHz}$ \\
\hline DC-link Vdc & $400 \mathrm{~V}$ \\
\hline Grid voltage Vg & $220 \mathrm{Vrms}$ \\
\hline Inverter-side filter inductance L1 & $4.2 \mathrm{mH}$ \\
\hline Inverter-side parasitic resistance $\mathrm{r} 1$ & $0.21 \Omega$ \\
\hline Grid-side filter inductance L2 & $1 \mathrm{mH}$ \\
\hline Grid-side parasitic resistance r2 & $0.04 \Omega$ \\
\hline Grid inductance Lg & $1.5 \mathrm{mH}$ \\
\hline Grid parasitic resistance rg & $0.09 \Omega$ \\
\hline Filter capacitor C & $7 \mu \mathrm{F}$ \\
\hline
\end{tabular}




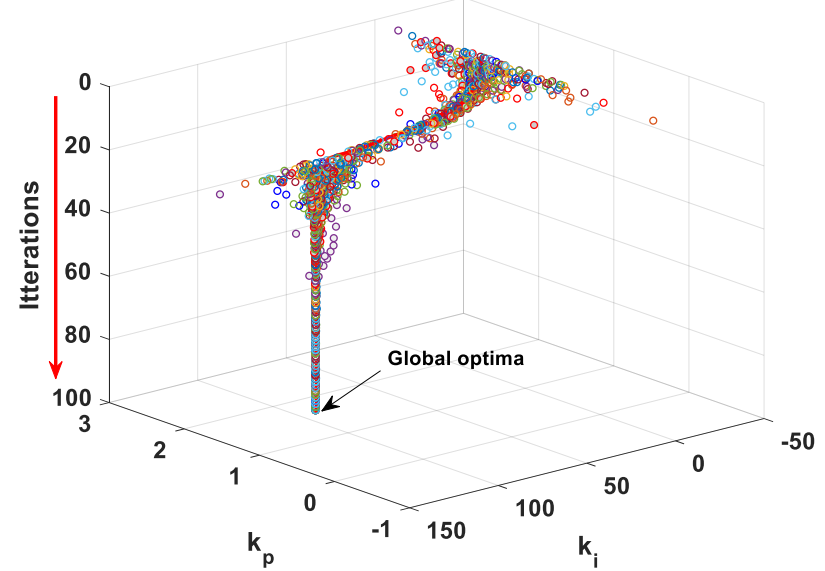

Figure.8 Particles convergence towards the global optima

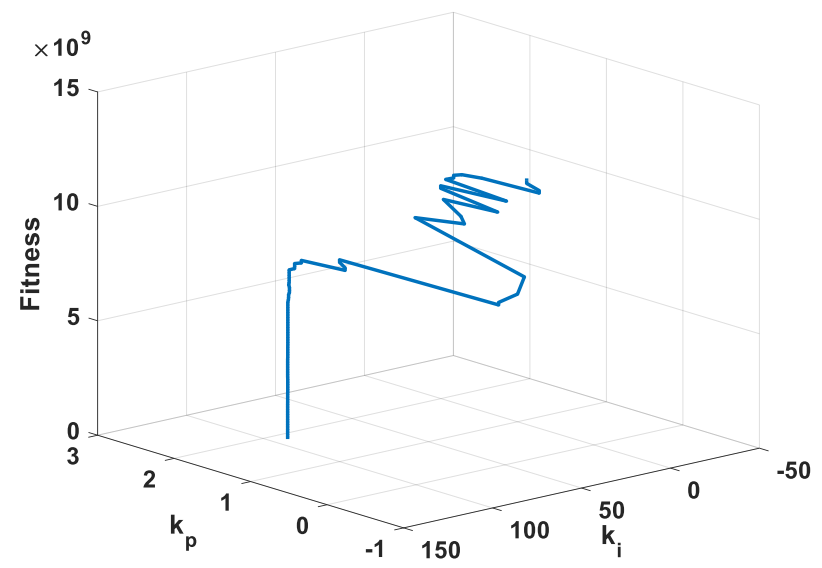

Figure.9 Fitness function minimization trajectory

The final position of the particles after 100 iterations illustrates the optimum values of the current controllers $\left(K_{p}\right.$ and $\left.K_{i}\right)$. Also, Fig.9 shows the trajectory of the objectives FF during the optimization process.

Furthermore, for results validation, the bodeplot and the root-locus of the open and closed-loop of the compensated system are shown in Fig.10 and Fig.11, from which it can be seen that the phase margin of the speed loop is about $M_{p}=87^{\circ}$ and the crossover frequency is $\omega_{c r}=230 \quad(\mathrm{rad} / \mathrm{s})$ with sufficient stability margin and damping ratio from 0.01-0.55. To recognize the effectiveness of the proposed algorithm a step response comparison between the proposed method and the time response method, (using RF and THD). Also, with the traditional simple trial and error method, presented in [20], are illustrated in Fig. 12.

Consequently, the overall LCL-filter gridconnected system is simulated using the obtained optimum controller as shown in Fig.13 the performance of the injected dq-current and active reactive power are illustrated in Fig.14 and Fig.15. Moreover, a comparison response between the proposed method and the traditional tuning method is depicted in Fig.16.

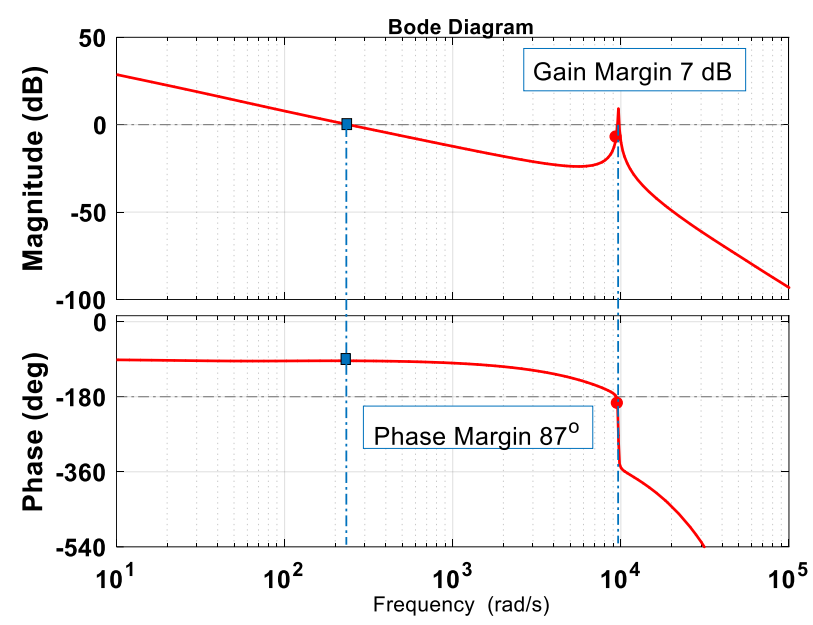

Figure.10 Bode-plot of the compensated system

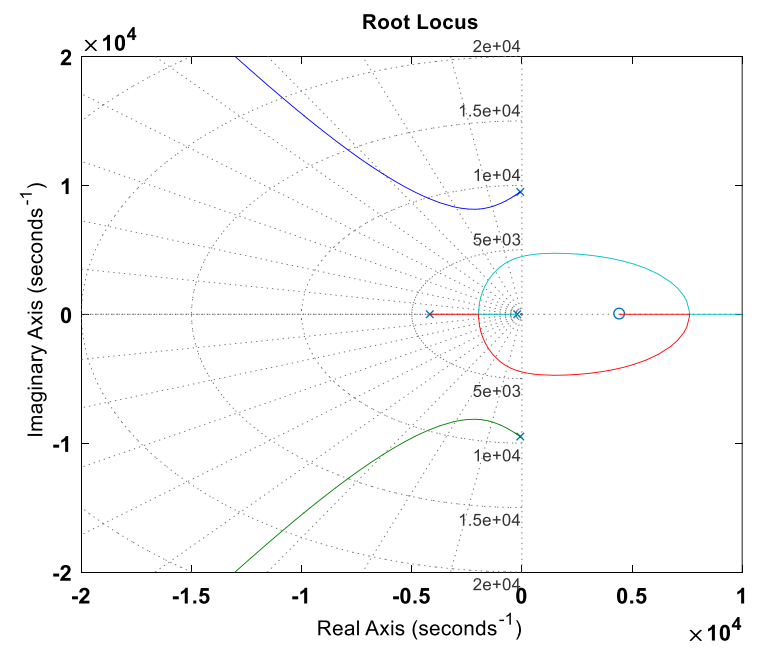

Figure.11 Pole-zero loci of the compensated system

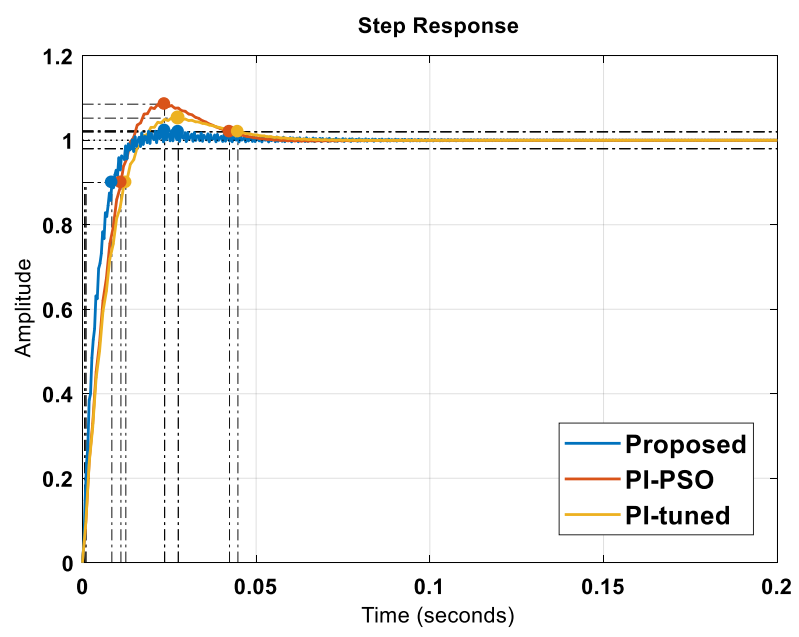

Figure.12 Comparison performance 


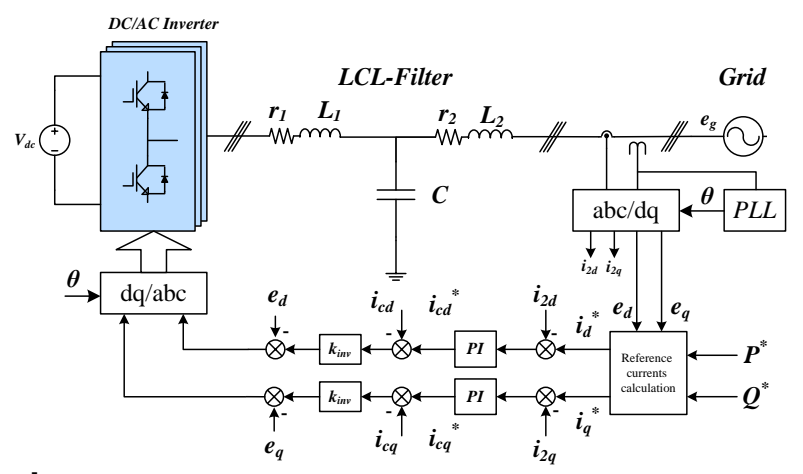

Figure. 13 The overall system simulation

The grid-injected current for different injected power can be seen in Fig.17. The FFT analysis of the grid-injected current can be shown in Fig.18. This validates that the injected current is a sinusoidal waveform, and the harmonics content of the LCL-filter output current is within the acceptable boundaries of international standards. Table 2 is a comparison study, lists the performance criteria, between the proposed method and the PIPSO method as well as the simple PI-tuned method were presented in [20] (by using the presented technique in this system).

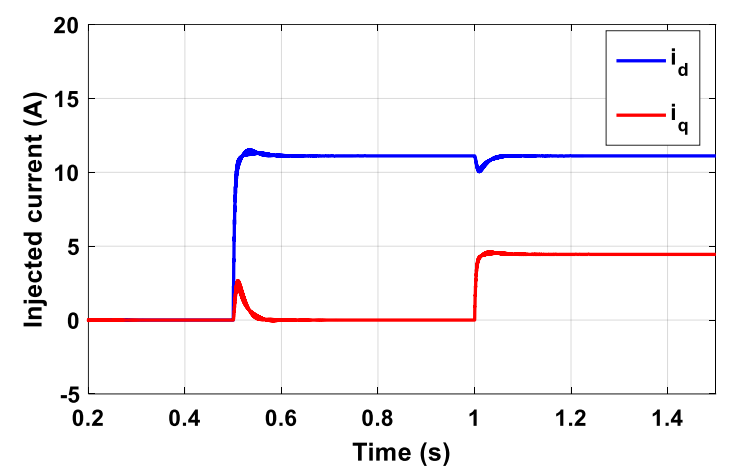

Figure. 14 Injected currents
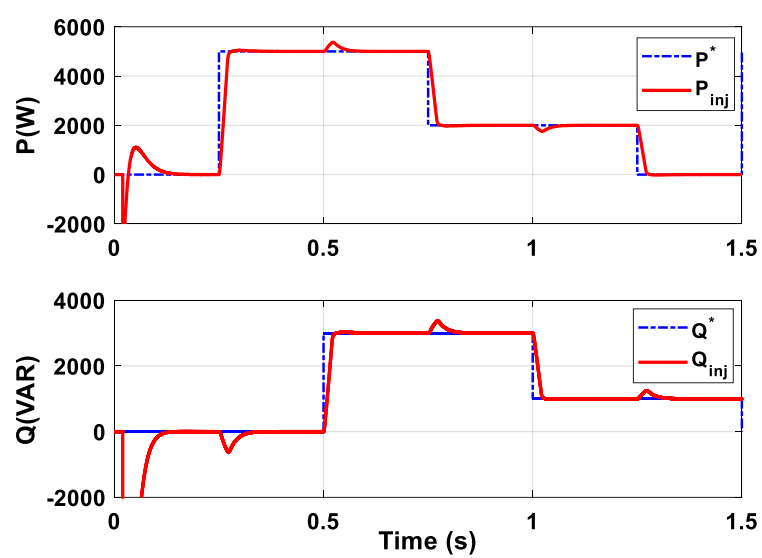

Figure. 15 Active and reactive injected powers
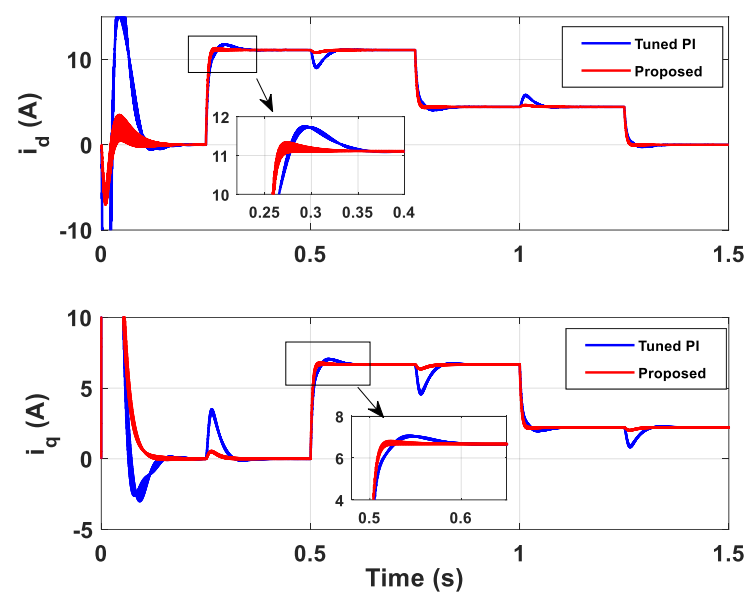

Figure. 16 Comparison responses

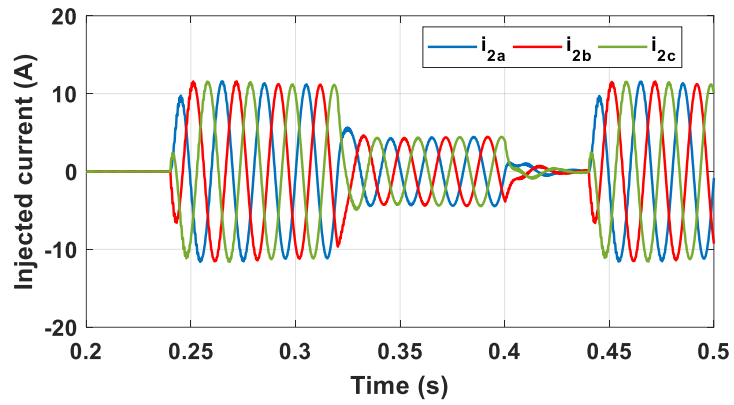

Figure.17 The grid-injected currents

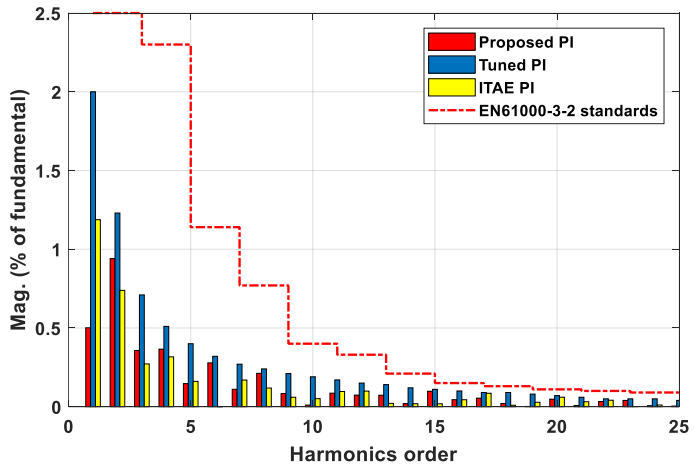

Figure.18 Comparison of harmonics contained

Table 2. Comparisons between different methods

\begin{tabular}{|l|c|c|c|}
\hline Criteria & Proposed & PI-tuned & PI-PSO \\
\hline Overshoot \% & 2.282 & 8.558 & 5.240 \\
\hline Rise time (ms) & 7.861 & 10.147 & 11.523 \\
\hline Settling time (ms) & 27.6 & 42.4 & 44.8 \\
\hline THD \% & 1.967 & 1.791 & 2.926 \\
\hline RF\% & 3.017 & 6.019 & 3.321 \\
\hline Gain margin (dB) & 7 & 6.8 & 7.1 \\
\hline phase margin (deg) & 87 & 62 & 85 \\
\hline Damping ratio & $0.01-0.55$ & $0.01-0.2$ & $0.01-0.45$ \\
\hline
\end{tabular}




\section{Discussions}

Simulation results verify that, however investigating time and frequency domain aspects simultaneously is a complicated method, the proposed optimization process of the current controller is an attractive procedure. Also, the concept of the system stability will be guaranteed and the system evolves more reliable. Fig.7 and Fig. 8 reveal the concentration of the location of the particles and the analogous fitness function approaching the global optimum position. Precisely, the collecting of the most particles on the global optima during a few iterations confirms the effectiveness of the optimization process and the objective function. As compared with other works, that expects a considerable number of iterations to achieve the global optima, which decreases the computation time. Besides, the bode plot and rootlocus diagrams for open-loop and closed-loop show that the system has adequate phase and gain margins with a fast time response of the dominant poles, as shown in Fig.10 and Fig.11 respectively. Those margins extend the stability boundaries of the controller against gains variation. The step responses of the dq-current and power controllers depicted in Fig.14, Fig.15 and Fig.16 show excellent performances of following different increasing decreasing reference commands. Besides, the harmonics level of the grid-injected current meets the standard requirements as in EN61000-3-2 and IEEE1547 as shown in Fig.18. Obviously, the proposed controller design shows superior performance over that of the previous works as recorded in Table 2.

\section{Conclusions}

This paper presents a procedure for tuning the PI controller of the LCL-filter grid-connected system. This includes an optimization technique to searching for the optimal parameters of the PI controller. The method depends on investigating the behavior of the system in both time and frequency domains. Moreover, the method combines these constraints with the Routh-Hurwitz's stability criteria to increase the effectiveness of the optimization process. Simulations results demonstrate the effectiveness of the proposed tuning method by performing a high degree of stability $\left(M_{p}=87^{\circ}\right.$, $M g=7 \mathrm{~dB}$, and damping ratio=0.55) at the worst case of gain variation. Combining the Routh-Hurwitz's theorem with the PSO routine points to a powerful optimization rule by eliminating the ineffective particles that return undesired performance as well that may collapse the stability criteria. Results demonstrate the excellent realization of the presented method (rise time $=7.861 \mathrm{~ms}$, settling time $=27.6 \mathrm{~ms}, \quad$ overshoot $=2.282 \%, \quad \mathrm{THD}=1.967 \%$, and $\mathrm{RF}=3.017 \%$ ), comparing with former tuning techniques. Considering the time and frequency responses supported by stability certification of Routh-Hurwitz's theory. Results prove that the proposed procedure is an interesting method might be extended, in future work, to include the applications of controller design for nonlinear or parameter variation systems.

\section{Conflicts of Interest}

The authors declare there is no conflict of interest. There are not any personal circumstances or interest that may be perceived as inappropriately influencing the representation or interpretation of reported research results.

\section{Author Contributions}

Conceptualization, Fadhil and Lina; methodology, Fadhil; software, Fadhil; validation, Fadhil, Lina, and Abdulrahim; formal analysis, Fadhil; investigation, Fadhil; resources, Fadhil, Lina, and Abdulrahim; data curation, Fadhil, Lina, and Abdulrahim; writing - original draft preparation, Fadhil; writing - review and editing, Fadhil; visualization, Fadhil; supervision, Fadhil; project administration, Fadhil; funding acquisition, Fadhil, Lina, and Abdulrahim.

\section{References}

[1] Y. He, H. Chung, C. Lai, X. Zhang, and W. Wu, "Active Cancelation of Equivalent Grid Impedance for Improving Stability and Injected Power Quality of Grid-Connected Inverter Under Variable Grid Condition", IEEE Transactions on Power Electronics, Vol. 33, No. 11, pp. 9387-9398, 2018.

[2] Y. Gui, Q. Xu, F. Blaabjerg, and H. Gong, "Sliding mode control with grid voltage modulated DPC for voltage source inverters under distorted grid voltage", CPSS Transactions on Power Electronics and App., Vol. 4, No. 3, pp. 244-254, 2019.

[3] W. Choi, W. Lee, D. Han, and B. Sarlioglu, "New Configuration of Multifunctional GridConnected Inverter to Improve Both CurrentBased and Voltage-Based Power Quality", IEEE Transactions on Industry Applications, Vol. 54, No. 6, pp. 6374-6382, 2018.

[4] L. Jia, X. Ruan, W. Zhao, Z. Lin, and X. Wang, "An Adaptive Active Damper for Improving 
the Stability of Grid-Connected Inverters Under Weak Grid", IEEE Tran. On Power Electron, Vol. 33, No. 11, pp. 9561-9574, 2018.

[5] S. Yoon and K. Kim, "Multi-loop current control for an inductive-capacitive-inductivefiltered grid-connected inverter with frequencyadaptive capability under distorted grid environment", IET Power Electronics, Vol. 12, No. 6, pp. 1521-1531, 2019.

[6] X. Zhang, D. Xia, Z. Fu, G. Wang, and D. Xu, "An Improved Feedforward Control Method Considering PLL Dynamics to Improve Weak Grid Stability of Grid-Connected Inverters" IEEE Transactions on Industry App., Vol. 54, No. 5, pp. 5143-5151, 2018.

[7] L. Wang, C. Lam, and M. Wong, "Multifunctional Hybrid Structure of SVC and Capacitive Grid-Connected Inverter (SVC//CGCI) for Active Power Injection and Non-active Power Compensation", IEEE Transactions on Industrial Electronics, Vol. 66, No. 3, pp. 1660-1670, 2019.

[8] H. Goh, M. Armstr, and B. Zahawi, "Adaptive control technique for suppression of resonance in grid-connected PV inverters", IET Power Electronics, Vol. 12, No. 6, pp. 1479-1486, 2019.

[9] X. Li, J. Fang, P. Lin, and Y. Tang, "Active Magnetic Decoupling for Improving the Performance of Integrated LCL-Filters in GridConnected Converters", IEEE Transactions on Industrial Electronics, Vol. 65, No. 2, pp. 1367-1376, 2018.

[10] X. Zhou, "Robust Grid-Current-Feedback Resonance Suppression Method for LCL-Type Grid-Connected Inverter Connected to Weak Grid", IEEE Journal of Emerging and Selected Topics in Power Electronics, Vol. 6, No. 4, pp. 2126-2137, 2018.

[11] X. Wu, L. Zuo, J. Yi, and L. Han, "A double closed-loop control strategy for LCL-type gridconnected inverter based on combination state feedback", In: Proc. of 2016 International Conference on Smart Grid, Chengdu, pp. 359363, 2016.

[12] Y. He, Y. Peng, B. Wangm and L. Hang, "Master-slave current regulation of an LCLfilter-based grid-connected inverter under variable grid condition", The Journal of Eng., Vol. 2019, No. 18, pp. 4896-4899, 2019.

[13] Y. He, B. Wang, X. Xie, L. Shen, and P. Zeng, "A Decoupled Hybrid Current Control for Improving the Performance of $60^{\circ}$ DPWMBased Three-Phase Grid-Connected Inverter", IEEE Access, Vol. 8, pp. 876-888, 2020.
[14] H. Zhang, J. Xian, J. Shi, S. Wu, and Z. Ma, "High Performance Decoupling Current Control by Linear Extended State Observer for Three-Phase Grid-Connected Inverter With an LCL Filter", IEEE Access, Vol. 8, pp. 1311913127, 2020.

[15] M. Wang, X. Wang, J. Qiao, and L. Wang, "Improved Current Decoupling Method for Robustness Improvement of LCL-type STATCOM Based on Active Disturbance Rejection Control", IEEE Access, Vol. 7, pp. 121781-121792, 2019.

[16] V. Kipke, J. Chhor, and C. Sourkounis, "Actively Damped PI-based Control Design of Grid-Connected Three-Level VSC with LCL Filter", In: Proc. of IECON 2018 - 44th Annual Conference of the IEEE Industrial Electronics Society, Washington, DC, pp. 973-978, 2018.

[17] X. Tang, W. Chen, and M. Zhang, "A Current Decoupling Control Scheme for LCL-Type Single-Phase Grid-Connected Converter", IEEE Access, Vol. 8, pp. 37756-37765, 2020.

[18] C. S. Lim, S. S. Lee, Y. C. C. Wong, I. U. Nutkani, and H. H. Goh, "Comparison of Current Control Strategies Based on FCS-MPC and D-PI-PWM Control for Actively Damped VSCs With LCL-Filters", IEEE Access, Vol. 7, pp. 112410-112423, 2019.

[19] R. Teodorescu, M. Liserre, and P. Rodriguez, Grid Converters for Photovoltaic and Wind Power Systems, Chichester, United Kingdom: John Wiley \& Sons, 2011.

[20] S. Fahad, A. J. Mahdi, W. H. Tang, K. Huang, and Y. Liu, "Particle Swarm Optimization Based DC-Link Voltage Control for Two Stage Grid Connected PV Inverter", In: Proc. of 2018 International Conference on Power System Technology, Guangzhou, pp. 2233-2241, 2018.

[21] A. G. Haddad, K. Al-Wahedi, and A. Al-Durra, "PSO-Based LQR Design for Grid-Connected LCL Filter with THD Constraints", In: Proc. of IECON 2019 - 45th Annual Conference of the IEEE Industrial Electronics Society, Lisbon, Portugal, pp. 1962-1966, 2019.

[22] Q. N. Trinh, P. Wang, Y. Tang, and F. H. Choo, "Mitigation of DC and Harmonic Currents Generated by Voltage Measurement Errors and Grid Voltage Distortions in Transformer less Grid-Connected Inverters", IEEE Transactions on Energy Conversion, Vol. 33, No. 2, pp. 801$813,2018$.

[23] J. He and Y. W. Li, "Generalized Closed-Loop Control Schemes with Embedded Virtual Impedances for Voltage Source Converters with LC or LCL Filters", IEEE Trans. on 
Power Elect., Vol. 27, No. 4, pp. 1850-1861, April 2012.

[24] F. Liu, Y. Zhou, S. Duan, J. Yin, B. Liu, and F. Liu, "Parameter Design of a Two-Current-Loop Controller Used in a Grid-Connected Inverter System With LCL Filter", IEEE Transactions on Industrial Electronics, Vol. 56, No. 11, pp. 4483-4491, 2009.

[25] Y. Shi and R. Eberhart, "A modified particle swarm optimizer", In Proc. IEEE Int. Conf. Evol. Comput., Anchorage, AK, pp. 69-73, 1998. 